This item was submitted to Loughborough's Research Repository by the author.

Items in Figshare are protected by copyright, with all rights reserved, unless otherwise indicated.

\title{
Boustrophedon coverage path planning for UAV aerial surveys in wind
}

PLEASE CITE THE PUBLISHED VERSION

https://doi.org/10.1109/ICUAS.2017.7991469

PUBLISHER

(C) Institute of Electrical and Electronics Engineers (IEEE)

VERSION

AM (Accepted Manuscript)

LICENCE

CC BY-NC-ND 4.0

REPOSITORY RECORD

Coombes, Matthew, Wen-Hua Chen, and Cunjia Liu. 2018. "Boustrophedon Coverage Path Planning for UAV Aerial Surveys in Wind". figshare. https://hdl.handle.net/2134/28039. 


\title{
Boustrophedon Coverage Path Planning for UAV Aerial Surveys in Wind
}

\author{
Matthew Coombes, Wen-Hua Chen, Cunjia Liu \\ Department of Automotive and Aeronautical Engineering \\ Loughborough University, Loughborough, LE11 3TQ UK \\ Email: \{M.J.Coombes@lboro.ac.uk,W.Chen@lboro.ac.uk, C.Liu5@lboro.ac.uk\}
}

\begin{abstract}
In the quickly developing world of UAV remote sensing in precision agriculture, there is a need for a mission planning algorithm that accounts for the wind's effect on the survey. This paper presents a method to define a Boustrophedon aerial survey path in wind, for a given convex polygon, at a given sweep angle. It is shown that there exists no easy way to define a sweep angle relative to the wind that minimises flight time. This method is validated by comparing the numerical simulated path and times with a number of surveys run in the high fidelity X-Plane simulator.
\end{abstract}

Keywords-Coverage Path Planning; UAVs; Trochoids

\section{INTRODUCTION}

If the global population continues to rise at its current pace, the World Bank says we'll need to produce 50\% more food by 2050 to keep up with demand. Unfortunately, there is very little suitable land left too cultivate. In order to substantially keep up with this demand, land needs to be farmed more intensively and efficiently. In order to achieve this, modern technology needs to be utilised to farm more intelligently, in what is called precision agriculture.

Sensor and computer technology is advancing at an incredibly fast pace, over the years this has given farmers access to increasing amounts of data regarding their fields. For example; soil $\mathrm{Ph}$, water saturation, weeds, disease, crop health, and yield. This coupled with emerging analytic technologies, enables the data to be analysed and actionable information provided. Traditionally, data has to be gathered manually by agronomists by discretising the field and taking samples from each segment. Farms can be very large (Average US farm 175 ha [1]), and gathering data across the whole farm can be very time consuming and expensive. However there is an alternative to manual data gathering called remote sensing. This is when measurements of subject can be made at a distance from it. In the context of farming, this is done by analysing aerial imagery across a number of spectral frequencies. This was first made possible in 1972 when Landsat 1 was launched with a imaging sensor package capable of measuring in Red, Green, and two Inferred frequencies. However satellite imagery is low resolution, for example Landsat 1 had a spacial resolution of $79 \mathrm{~m} /$ pix. A more modern satellite like WorldView-2 that launched in 2009 has a resolution of $0.5 \mathrm{~m} /$ pix [2]. Satellite imagery has been used in multiple sensing applications for example; yield calculation [3], weed mapping [4], soil moisture content [5]. These sort of resolutions are adequate for some applications, but some require centimetre Ground Sample Distances (GSD), which satellites can not provide. Satellites have a low persistence, as their orbit may not pass over the target area for some time, and images are easily blocked by cloud, and haze. A solution is to collect images from a manned aircraft as they can fly under clouds, and as photos will be taken at lower altitudes, the GSD will be lower to see the finer details needed. However these sort of surveys are very expensive and are still constrained by weather and operational concerns.

The advances in MEMS sensors, and embedded computing has enabled small UAVs to become cheaply and widely available. By fixing a small imaging system to either a small fixed or rotary wing platform, this enables cheap remote sensing at low altitudes. As they will be flying anywhere from the ground level to $120 \mathrm{~m}$, the GSD's of $1-10 \mathrm{~cm}$ are easily achievable. They do not suffer from the same restrictive operational requirements of a manned aircraft, so can be flown much more frequently, to enable more detailed temporal studies of crops and farming. A good introduction to UAV remote sensing can be found in [6]. However a single image from low altitude will only cover a small area. By taking a number of images across a Region of Interest (ROI), they can be stitched together using Structure from motion photogrammetry software in to a geo-referanced orthomosaic or Digital Elevation Model (DEM) which will give the desired GSD over the whole area [7]. Images need to be localised relative to one another using image processing. Stitching them together requires that adjacent photos have enough overlap in order for the images to share enough image features to calculate their alignment as seen in Fig. 1. This means that the aircraft needs to be flown in a survey flight pattern over certain points in order to take enough photos with enough overlap to get a quality orthophoto or a DEM of the ROI.

In order to determine the survey path for the aircraft to follow, a path planner is needed which will generate a path to ensure complete photo coverage of the ROI. Small UAV will have a low mass and low airspeeds, which make them very susceptible to the effects of wind. This is clearly shown in [8] where wind added around 6 mins to a 21 min simulated fixed wing survey of an $1 \times 0.6 \mathrm{~km}$ area in a number of different sweep angles. Since small UAV's have limited endurance and range, optimising the path in wind is essential.

This type of planning is called Coverage Path Planning (CPP), which is the task of generating a path that passes over all points of an area of interest. This area is normally represented by a polygon. CPP has been extensively studied in literature for use in a range of applications, for example lawn mowing, milling [9], cleaning robots [10], agricultural field machines [11], rotary wing surveys [12], and fixed wing surveys [13]. 


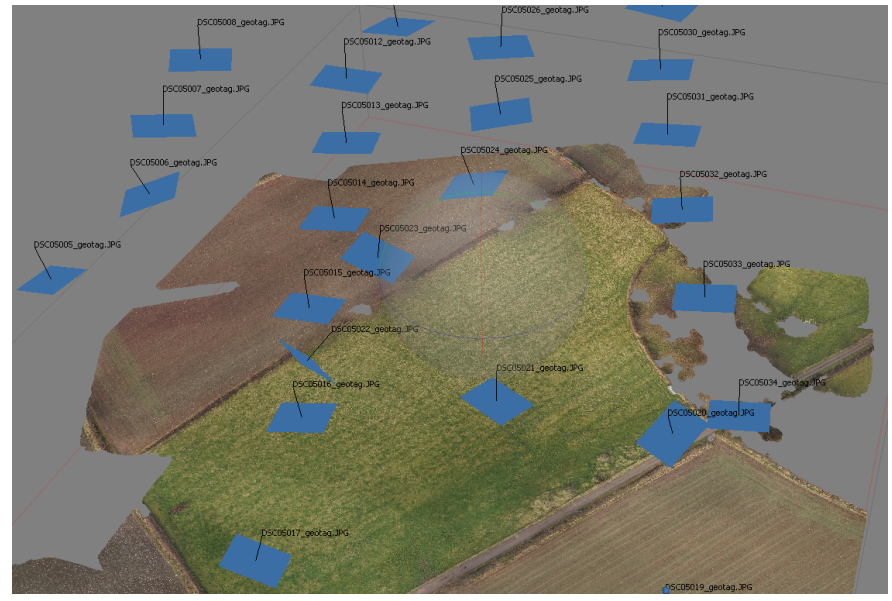

Fig. 1: Structure from motion photogrammetry software Agisoft's estimation of camera locations, and generated 3D model

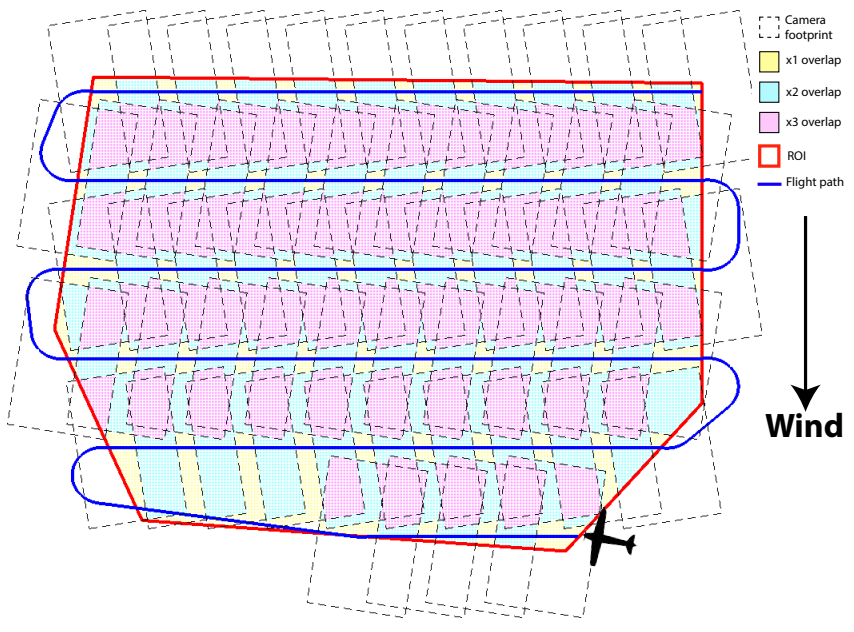

Fig. 2: Example Boustrophedon path of a convex polygon, showing the camera image footprints giving complete coverage. Sidelap $40 \%$, overlap $40 \%$

The simplest path that guarantees coverage of an area is a simple back and forth motion of the vehicle along the long axis of the polygon. This is called a Boustrophedon path, which means "way of the ox". This is a simple and effective path when performed within a simple convex polygonal area [ [14], [8]]. All that is required to construct this path is the ROI polygon, the sweep angle of the paths, and cross track spacing determined by the UAVs image footprint, and required lateral overlap. Fig. 2 shows an example of a Boustrophedon path displaying the footprint of each photo and demonstrating that the whole ROI is covered.

Most literature assumes that the path following for the vehicle is perfect, which for ground robots is close to true. However as fixed wing aircraft are more susceptible to external disturbances like wind, and have to maintain forward motion to stay in the air, this means that accurate path following is made much more difficult. This is why in [13] the sweep method is not used but a method which guides the aircraft to areas which have not been photographed adequately using a camera model. While this ensured total coverage, the flight time was much longer and required the camera to be gimbaled, as the flight path involved many turns.

Farm fields can have complex concave shapes, however Boustrophedon paths are inadequate for concave polygons. By employing the Boustrophedon method on a concave polygon, sections of the path could be outside of the polygon. While a UAV can pass over the polygon boundary as it is flying, this is inefficient as travel time outside is wasted. This is why a number of papers use Boustrophedon Cell Decomposition (BCD) to separate the concave polygon into a minimum number convex polygons, then the Boustrophedon path is used to define paths for each individual cell. This method is detailed in [15] where the exact decomposition is performed using trapezoidal decomposition [16]. Then these will form an adjacency graph which is used to recombine some of them to make the minimum number convex polygons, called Boustrophedon cellular decomposition. There are other approximate cellular decomposition techniques but tend to break the polygon in to more cells than the exact cell decomposition techniques [ [17], [9]].

If a vehicle is able to maintain a constant ground velocity, then the path with the lowest number of turns will be shortest [ [15], [8]]. During the turn, the aircraft will not be able to collect any data, this is as turns are conducted just outside the ROI, and any photos taken in a turn will not be orthogonal to the ground. All the pre-planning methods that use some kind of BCD try to optimise based on turn number. The most common method to minimise turns is to align the sweep angles with the long axis of one of the decomposed cells, as this will increase the length of each leg of the path thus decreasing number of turns. However a fixed wing aircraft's ground speed, and time in a turn depend heavily on its airspeed and the wind direction and speed. This could mean that number of turns is an inadequate optermisation parameter for BCD optermisations, if wanting to account for wind. To this end we propose an method to extend Boustrophedon paths to factor in wind. With the aim of using the developed calculations to find if there is best angle to fly relative to the wind to minimise flight time. This will done by exploring factors effecting individual cell optermisation, then extending it to a simple two cell BCD example.

The remainder of this paper is organised as follows; section II details the construction of Boustrophedon Paths to ensure coverage of the ROI, section III discusses the effect of wind on survey path planning, section 6 describes how to define turn paths in wind, section IV, section V so some simulation results from X-Plane flight simulator. Finally section VI makes some concluding remarks, and discusses what future work needs to be conducted.

\section{CONVEX POLYGON BOUSTROPHEDON PATHS}

Boustrophedon paths consist of back and forth motions at a particular sweep angle across the ROI. Each of the straight sweep paths can be defined by their endpoints which will be refereed to as waypoints. These waypoints can be defined as the locations where the sweep lines intersect with the ROI polygon, as shown in Fig. 3. The waypoint, at which the aircraft joins the sweep path is defined as $\left[x_{f}, y_{f}\right]$ and the heading is $\psi_{o}$, and the waypoint at the end of the sweep is 


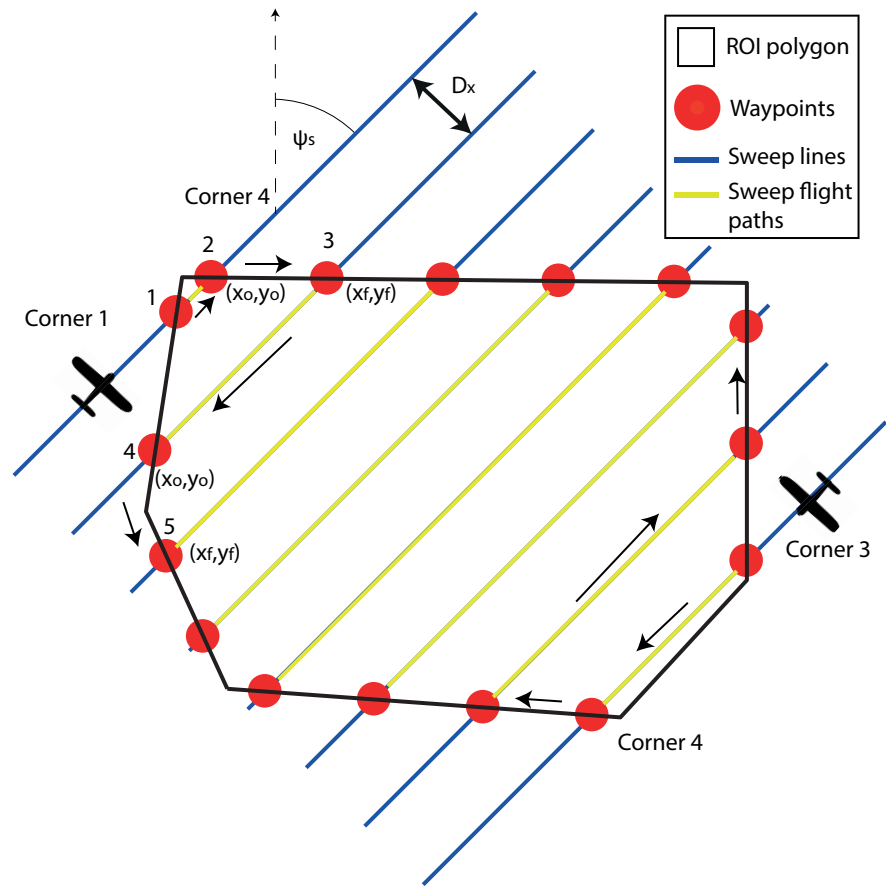

Fig. 3: Sweep line intersections with the ROI polygon define the straight line waypoints

$\left[x_{o}, y_{o}\right]$, at a heading of $\psi_{f}$. There are four corners that the aircraft can begin from, so the waypoints need to be put in the correct order for back and forth traversal.

The sweep angle $\left(\psi_{s}\right)$ of $45^{\circ}$ in Fig. 3 is clearly not the optimal angle as some of the straight paths are extremely short and so there are many more turns than are required. A method used in previous work is to align the sweep with the long axis of the polygon [15]. This can be done by calculating a minimum area bounding rectangle for the convex polygon, and calculating it's long axis angle $\left(\psi_{b b}\right)$ which can actually be directly calculated, which is shown in Fig. 4. For polygons with bounding rectangles that are close to square, will see less advantage from aligning the sweep angle to it's long axis.

To ensure that the whole ROI is completely photographed with the correct image overlap footprints, the back and forth paths have to be spaced correctly. The overlap is dictated by mission requirements, for example if high accuracy DEM's are needed a high overlap and sidelap \% of around $70 \%-$ $80 \%$, whereas if just orthophotos that are needed, this can be lowered to $50 \%-60 \%$. The GSD will be defined depending on the specific application, which in turn dictates the height to be flown, calculated below:

$$
h=\frac{N_{x} G S D}{\text { fov }_{h}}
$$

where $h$ is the height of the aircraft above the ground, GSD is in $\frac{m}{p i x}$, fov $_{x}$ is the horizontal angular field of view for the sensor, $N_{x}$ is the number of pixels the sensor has in the horizontal direction. Then the track spacing $D_{x}$ can be calculated as below:

$$
D_{x}=2 h \tan \left(\frac{f_{o v_{x}}}{2}\right)\left(1-w_{s}\right)
$$

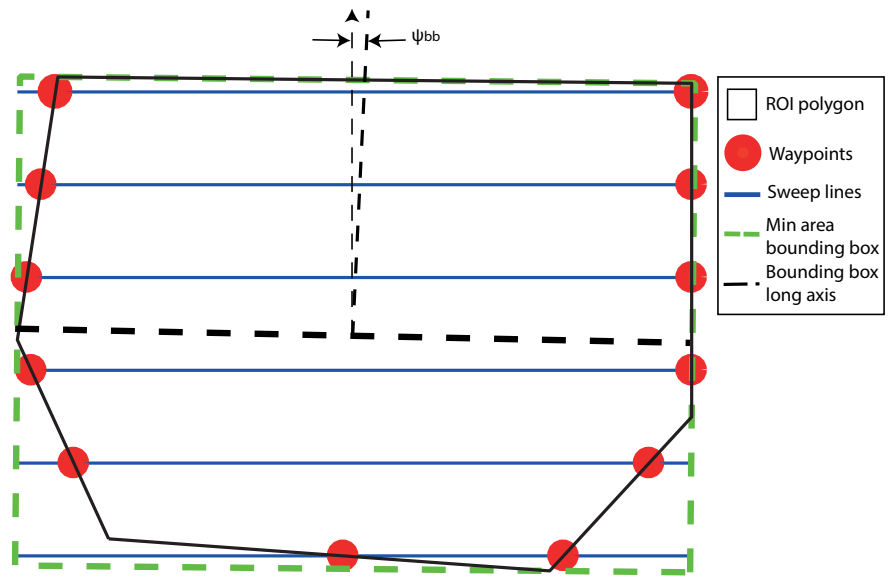

Fig. 4: Use of the minimum area bounding box of the ROI polygon to find sweep angle that achieves minimum number of turns

where $w_{s}$ is the desired image sidelap between tracks. This does however assume that the aircraft is facing perfectly orthogonal to the ground.

The frequency images need to be captured along each sweep path is dictated by overlap $w_{o}$. To calculate the distance between photo triggering points $D_{y}$ can be done with Eq. (2), but with the vertical FOV fov $v_{y}$. Due to camera processing times, and camera storage write speeds, the speed of image capture is limited to the cameras cycle time. For example the mirrorless DSLR Sony Nex 7 has a minimum cycle time of around $0.7 \mathrm{~s}$, onboard an aircraft travelling at $15 \mathrm{~m} / \mathrm{s}$ means that $D_{y}$ will have a minimum of $10.5 \mathrm{~m}$.

Between the straight sweep paths the aircraft needs to manoeuvre onto the next sweep. These turns alternate between right and left hand, each through an angle of $180^{\circ}$. This manoeuvre needs to be defined so to calculate and account for the extra distance and time taken in the turns. This can be achieved with Dubins paths. A Dubins path is the shortest curve that connects two points in a $2 \mathrm{D}$ plane with a constraint on the curvature (In this case maximum turn rate) [18]. Shown in Fig. 5 is a Dubins path that links the adjacent tracks to give a coherent flight path. The path is defined by two circles placed in relation to the waypoints with a radius defined by the aircraft's airspeed and maximum turn rate. Then all feasible internal and external common tangents are found between the two circles. In the case of Fig. 5 there is only one feasible external tangent. The tangent connects the two turn arcs along each circle to give a smooth path. As these are simple geometric shapes, and the aircraft is flying at a constant airspeed, makes calculating lengths and times simple.

\section{WIND}

Wind can effect a small aircraft significantly, the wind speed experienced by a small UAV can easily be $50 \%$ of the small UAVs airspeed. This is why on this scale of survey UAV's it is very important to account for wind. Wind will have a number of effects on a UAV survey. The ground speed between sweeps will be different, changing the flight time, as well as having an effect on the alignment of the images, discussed in Section III-A. In the turns 


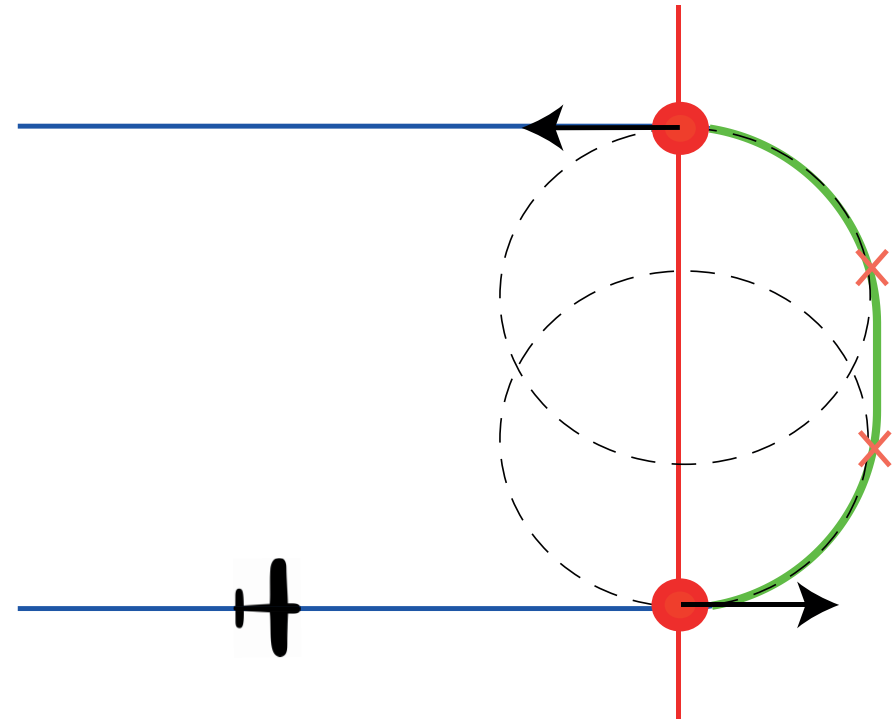

Fig. 5: Turn manoeuvre between sweeps in zero wind using Dubins Paths

Dubins curves assume zero wind, when performing the same manoeuvre in wind, the turn shape will be trochoidal not circular in the ground frame. To achieve the same minimum distance path the trochoidal turns are incorporated in to Dubins framework, this then gives a path length and time that accounts for the wind. This is discussed in Section 6.

\section{A. Wind on sweep paths}

To compensate for the wind along the straight sweep paths, the aircraft, instead of it heading being directly along the track, it has to point slightly into wind to ensure it's ground velocity vector is pointing along the track. This heading adjustment is called the Wind Correction Angle (WCA) $\left(\psi_{W C A}\right)$, which is calculated below. This can be derived from the wind triangle using the sine rule.

$$
\psi_{W C A}=\arcsin \left(\left(\frac{V_{w}}{V}\right) \sin \left(\psi_{s}-\psi_{w}\right)\right)
$$

where $\psi_{w}$ is the wind direction, $\psi_{s}$ is the sweep direction angle, $V$ and $V_{w}$ are airspeed and wind speed respectively. $\psi_{s}-\psi_{w}$ is the the aircraft's heading relative to the wind which as a critical variable is simplified and referred to as the Wind To Track Angle $\left(\psi_{w t a}\right)$.

$$
\psi_{w t a}=\psi_{s}-\psi_{w}
$$

Now the WCA is known the ground speed $\left(V_{g}\right)$ and sweep flight time can be calculated. $V_{g}$ is the sum of the $\mathrm{x}$-components velocity through the air mass and the $\mathrm{x}$ component of the wind velocity, shown below

$$
V_{g}=\left(V \cos \left(\psi_{W C A}\right)\right)+\left(V_{w} \cos \left(\psi_{w t a}\right)\right)
$$

The $\psi_{w c a}$ will also have the effect of rotating the image footprint as well. At low $\psi_{w c a}$ 's and high sidelap $\%$ this will most likely have no effect on coverage, but at high $\psi_{w c a}$ and low image sidelap the small gaps in coverage may appear.

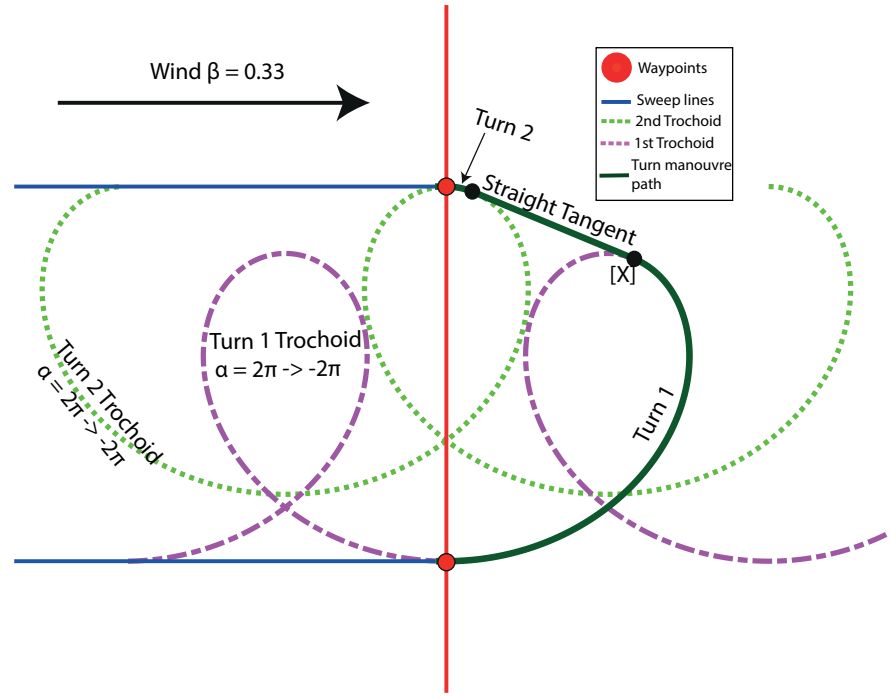

Fig. 6: Comparison between a left straight left Dubins path in nil wind to a trochoidal path in wind.

\section{B. Trochoidal turn paths}

When a circle is drawn in a steady moving frame of reference it becomes a shape called a trochoid. It was shown in previous literature that Dubins paths can be applied in wind by finding the feasible tangents between the two trochoids at the start and the end of the turn manoeuvre [19] and [20]. There are four different feasible paths, as the initial and final turn is can be either left (L) or right (R) hand turn connected with the straight (S) tangent, LSL LSR RSR RSL. However all turns in a Boustrophedon path will be LSL or RSR, as any other would mean that the aircraft would have to turn through more that $180^{\circ}$. This simplifies the problem, as same side turns have a full analytical solution, where opposite side turn calculations are transcendental so need a root finding technique to solve.

$\alpha \quad$ Trochoid reference angle

$\beta \quad$ Wind airspeed to airspeed ratio

$\psi_{f} \quad$ Final heading

$\psi_{w} \quad$ Wind direction

$\delta_{1} \quad$ Turn direction of first turn (+ve right hand turn)

$\delta_{2} \quad$ Turn direction of second turn (+ve right hand turn)

$\psi_{0} \quad$ Initial heading

$\dot{\psi} \quad$ Aircraft turn rate

$x_{0} \quad$ Initial position $\mathrm{x}$-axis

$y_{0} \quad$ Initial position $\mathrm{y}$-axis

$x_{f} \quad$ Final position $\mathrm{x}$-axis

$y_{f} \quad$ Final position y-axis

$D_{x} \quad$ Distance between sweep lines

Fig. 6 shows the same part of the Boustrophedon path as Fig. 5 however the circular turns have been replaced with trochoids to give a similarly smooth trajectory. Shown in Eq. (6) is the parametric equation for a trochoid. It has been rotated in order to put the equation in to the trochoidal frame with subscript $t$, this means the everything is rotated to make the $y$-axis in line with the direction of wind. 


$$
\begin{array}{r}
x_{t}=-R \cos (\alpha) \\
y_{t}=R \sin (\alpha)+R \beta \alpha
\end{array}
$$

The construction of these trochoidal turn paths can be performed by knowing the start $\left(x_{o}, y_{o}\right)$ and end point $\left(x_{f}, y_{f}\right)$, and the initial $\left(\psi_{o}\right)$ and final headings $\left(\psi_{f}\right)$ of the manoeuvre. Also with airspeed $(V)$, turn rate $(\dot{\psi})$ and wind speeds.

The calculations to achieve this are laid out in [19], but the critical calculations are repeated here for completeness. The reference angle $\alpha_{1}$, and $\alpha_{2}$ needs to be found, these are the reference angles for where the two trochoids shares a common tangent. This is calculated below

$$
\alpha=\arctan \left(\frac{\left(x_{t 2}-x_{t 1}\right)}{\left(y_{t 2}-y_{t 1}\right)+V\left(\frac{\eta_{1}-\eta_{2}+2 k \pi}{\delta_{2}}\right)}+2 \pi m\right)
$$

where $\left(x_{t 1}, y_{t 1}\right)$ is a translation of the first trochoid to $\left(x_{o}, y_{o}\right),\left(x_{t 2}, y_{t 2}\right)$ is the same but for the second trochoid defined in (14), and (15) respectively. Where $k, m \in$ $[-3,-2,-1,0,1,2]$. As there are in fact infinite repeating trochoids, $\mathrm{k}$ and $\mathrm{m}$ dictate which individual trochoids the tangent are found between. $\eta_{1}$ and $\eta_{2}$ are angles to rotate the trochoids to be inline with the wind, defined below

$$
\eta_{1}=\psi_{0}-\psi_{w}, \quad \eta_{2}=\psi_{o}-\psi_{w}-\delta_{2} 2 \pi
$$

where $\delta_{1}$, and $\delta_{2}$ are the turn direction of the trochoids, where +1 is a right turn, and -1 is a left turn.

$$
\begin{gathered}
x_{t 1}=\frac{V}{\delta_{1} \dot{\psi}} \cos \left(\eta_{1}\right)+x_{t 0} \\
y_{t 1}=-\frac{V}{\delta_{1} \dot{\psi}} \sin \left(\eta_{1}\right)+y_{t 0}
\end{gathered}
$$

where $\left(x_{t 0}, y_{t 0}\right)$ is $\left(x_{0}, y_{0}\right)$ rotated by $\psi_{w}$ into the trochoidal frame.

$$
\begin{array}{r}
x_{t 2}=\frac{V}{\delta_{2} \dot{\psi}} \cos \left(\eta_{2}\right)+x_{t f} \\
y_{t 2}=\frac{V}{\delta_{2} \dot{\psi}} \sin \left(\eta_{2}\right)+2 \frac{V}{\delta_{2} \dot{\psi}} \beta \pi+y_{t f}
\end{array}
$$

When $\alpha_{1}$ is found the equation below can be used to calculate $\alpha_{2}$

$$
\alpha_{1}=\frac{\delta_{1}}{\delta_{2}} \alpha_{1}+\frac{\eta_{1}-\eta_{2}+2 k \pi}{\delta_{2}}
$$

1) Time and length calculations: Now the points of tangency have been defined with $\alpha_{1}$, and $\alpha_{2}$, the flight time, and distance in this manoeuvre can be calculated.

The time and distance travelled during this manoeuvre are derived in [20]. To find the distance travelled around a trochoid elliptic integrals of the second kind are used below

$$
L_{1}=2 R(1+\beta) E\left(\frac{\alpha_{1}}{2} \mid \frac{4 \beta}{(1+\beta)^{2}}\right)-2 R(1+\beta) E\left(0 \mid \frac{4 \beta}{(1+\beta)^{2}}\right)
$$

where $L_{1}$ is the arc length subtended by the aircraft around the first trochoid.

$L_{2}=R(1+\beta) E\left(2 \pi \mid \frac{4 \beta}{(1+\beta)^{2}}\right)-2 R(1+\beta) E\left(\frac{\alpha_{2}}{2} \mid \frac{4 \beta}{(1+\beta)^{2}}\right)$

To find the length of the straight portion of the manoeuvre the points of tangency on both trochoids $\left(x_{t P 1}, y_{t P 1}\right)$, $\left(x_{t P 2}, y_{t P 2}\right)$ need to be found, using the calculated $\alpha_{1}$, and $\alpha_{2}$, shown below

$$
\begin{array}{r}
x_{t P 1}=-\frac{V}{\delta_{1} \dot{\psi}}\left(\cos \left(\alpha_{1}+\eta_{1}\right)+\cos \left(\eta_{1}\right)\right)+x_{t 0} \\
y_{t P 1}=\frac{V}{\delta_{1} \dot{\psi}}\left(\sin \left(\alpha_{1}+\eta_{1}\right)+\beta \alpha_{1}+\sin \left(\eta_{1}\right)\right)+y_{t 0}
\end{array}
$$

and for the final trochoid in:

$$
\begin{array}{r}
x_{t P 1}=\frac{V}{\delta_{2} \dot{\psi}}\left(\cos \left(\eta_{2}\right)-\cos \left(\alpha_{2}+\eta_{2}\right)\right)+x_{t f} \\
y_{t P 1}=\frac{V}{\delta_{2} \dot{\psi}}\left(\sin \left(\alpha_{2}+\eta_{2}\right)+\beta \alpha_{2}+\sin \left(\eta_{2}\right)+2 \beta \pi\right)+y_{t f}
\end{array}
$$

By adding the lengths of both trochoids and the distance between the two points of tangency, the whole flight distance in the turn manoeuvre can be found.

$$
L=L_{a}+L_{b}+\sqrt{\left(y_{t P b}-y_{t P a}\right)^{2}+\left(x_{t P b}-x_{t P a}\right)^{2}}
$$

The flight time for the first trochoid $\left(t_{1}\right)$ is easily calculated from the angle subtended and the known turn rate of the aircraft.

$$
t_{1}=\frac{V}{R} \alpha_{1}
$$

Where the time in the second turn $\left(t_{2}\right)$ is defined

$$
t_{2}=\frac{2 \pi}{\dot{\psi}}-t_{a}-\frac{\eta_{1}-\eta_{2}+2 k \pi}{\delta_{2} \dot{\psi}}
$$

\section{NumERICAL RESUlTS}

To show the effect wind has on a survey, and to investigate the time optimum sweep angle relative to the wind $\left(\psi_{t} s\right)$, a numerical Monte Carlo simulation is run. This is performed across a range of $\psi_{t} s$, for a circular ROI polygon. By defining the survey ROI polygon as a circle, the number of sweeps and their length will remain unchanged at any sweep angle, an example is shown in Fig. 7.

In the simulation the ROI is a circle with a $1 \mathrm{~km}$ radius, the wind is northerly $\left(\psi_{w}=0\right)$. Surveys will be conducted across six wind speeds, giving $\beta$ 's from 0 to the very extreme 0.5 . At each wind speed 36 surveys are calculated for relative sweep angles $\psi_{t s} 0-360^{\circ}$ in increments of $10^{\circ}$ (180-360 is identical to 0-180). The flight distance and times for these surveys are shown in Fig. 8. The flight and photogrammetry parameters are shown below:

$$
\begin{array}{lll}
\psi_{w}=0^{\circ} & V=20 \mathrm{~m} / \mathrm{s} & \dot{\psi}=0.7 \mathrm{rad} / \mathrm{s} \\
D_{x}=337 \mathrm{~m} & D_{y} & w_{s}=0.4 \\
w_{o}=0.4 & G S D=0.08 \frac{\mathrm{m}}{\mathrm{pix}} & h=250 \mathrm{~m}
\end{array}
$$

It can be seen that the flight time is significantly effected by the wind, with a total survey time penalty of 100 s for $10 \mathrm{~m} / \mathrm{s}$ wind to nil wind at $\psi_{t s}$ of $270^{\circ}$. It also seems that $\psi_{t s}$ of $270^{\circ}$ minimises the time for these flight conditions. Curiously at $90^{\circ}$ there is no flight time minimum even though the survey is still perpendicular to the wind. There 


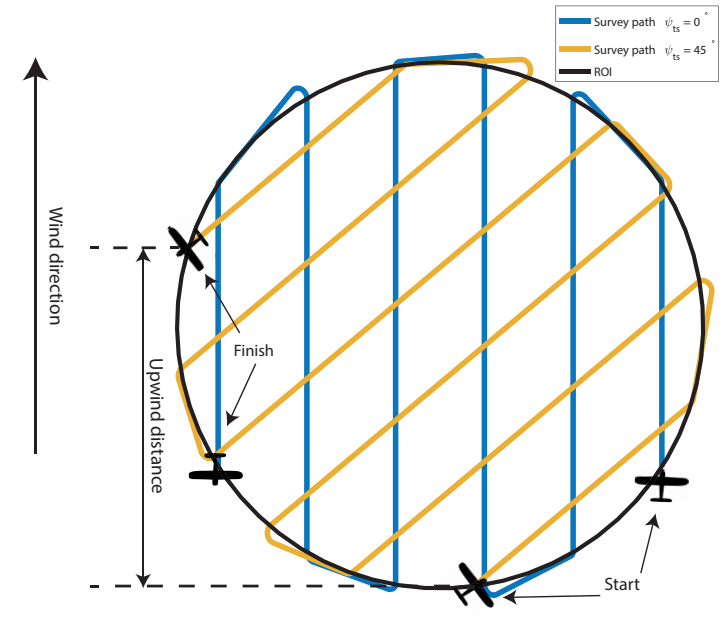

Fig. 7: Example of two surveys at different sweep angles on a circular ROI

are two factors that effect the flight time: Flight time in the sweeps, and flight time in the turns.

The only variable that will effect time in the sweeps is the ground speed of the aircraft, as the distance is fixed. Shown in Fig. 9 is the time along the sweep portion of the flight. It shows that a sweep angle at $270^{\circ}$ and $90^{\circ}$ to the wind the sweep time is minimised. This is due to the progressively slower aircraft $V_{g}$ along the sweeps due to an increasing $W C A$, pointing the aircraft further into wind keeping it along the track.

The second factor is the time to conduct the trochoidal turns. This is very non-linear as there are a huge number of factors governing the time to fly around all the turns. The shape and ground speed profile will change at different $\psi_{t s}$. Fig. 10 shows that the turning flight time has a complex relationship with sweep angle, where it is minimised at around $260^{\circ}$, and maximum at around $70^{\circ}$. Where the time difference between those points is around 60 s for a $V w$ of 6 $\mathrm{m} / \mathrm{s}$. The distance plot in Fig. 8 effectively shows the change in turn distance (as the sweep distance is fixed). The total distance difference is about $300 \mathrm{~m}$ for a $V w$ of $6 \mathrm{~m} / \mathrm{s}$ which only accounts for approximately $15 \mathrm{~s}$ of extra time from the extra distance. The further $45 \mathrm{~s}$ will be accounted for in the difference in $V_{g}$ due to the wind helping or hindering the aircraft between its start and finish point.

How far the start and finish points are upwind or downwind from each other is dictated by the starting corner and the $\psi_{t s}$, as demonstrated in Fig. 7. If an aircraft starts upwind from the survey finish point it will be helped as it will have a greater tail wind, and vice versa. Fig. 11 shows this distance for all sweep angles in the simulation. It can clearly be seen that the aircraft is the furthest upwind at around $250^{\circ}$, and furthest downwind at $70^{\circ}$. Shown in Fig. 10 is the total flight time spent in the turning portion of the survey, the minimum and maximum points on the curve concide with the maximum and minimum points in Fig. 11.

In Fig. 10 you would expect to see symmetry around $180^{\circ}$ if the upwind distance was the only contributing factor. It is not, the other influence is a turn distance increase at $90^{\circ}$, and decrease at $270^{\circ}$ (as shown in Fig. 8). This distance increase is simply due to the wind squeezing the flight path shorter
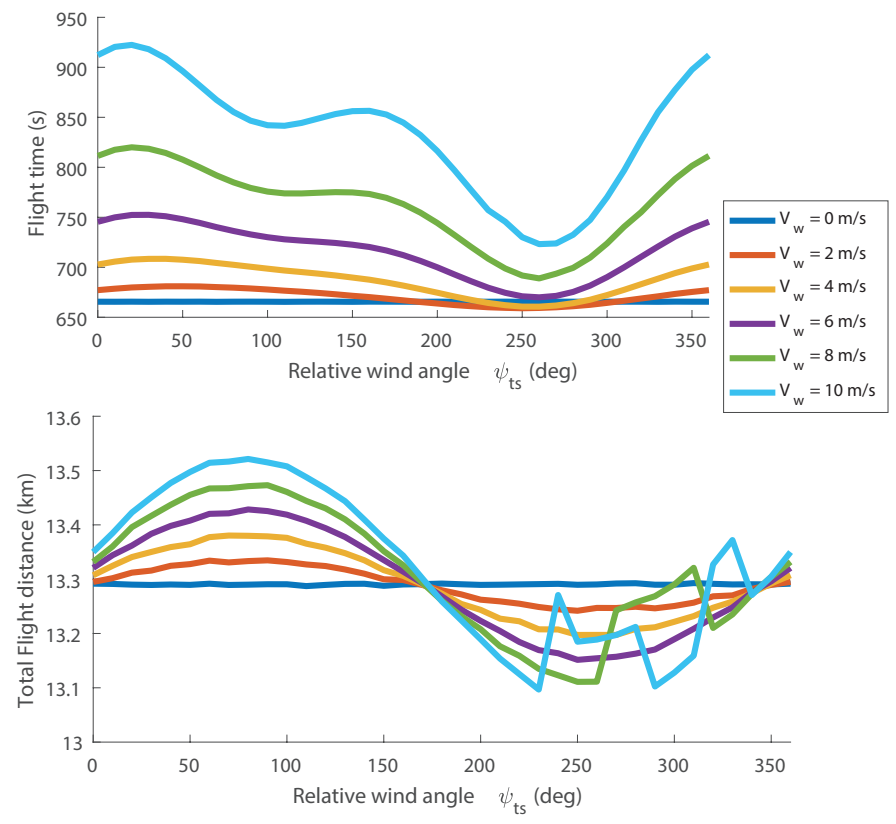

Fig. 8: Flight time (top) Total path distance (bottom) plots for $0-360^{\circ} \psi_{t s}$ for a $1 \mathrm{~km}$ radius circular ROI

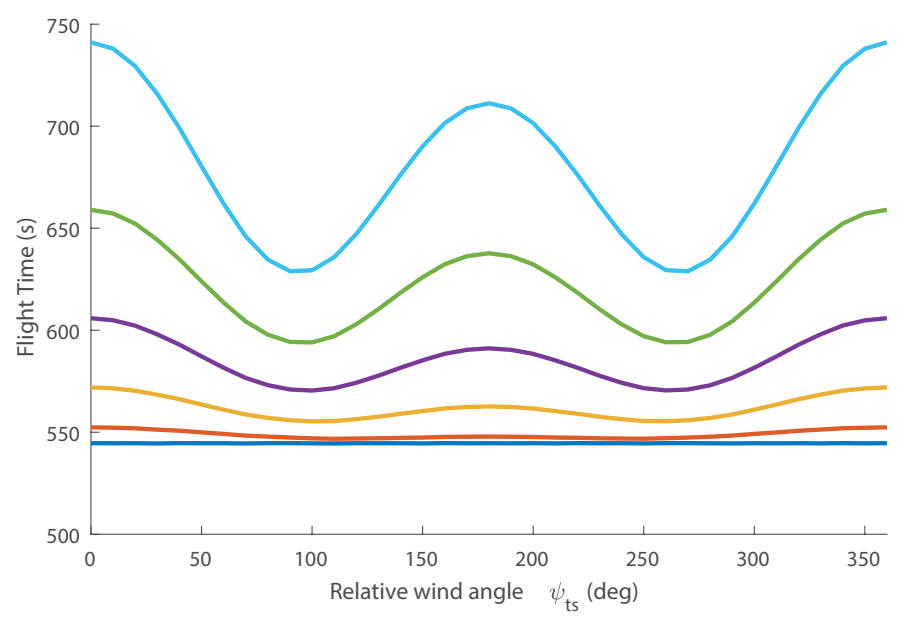

Fig. 9: Total flight time along sweep paths for $0-360^{\circ} \psi_{t s}$, at $V_{w}$ for $0-10 \mathrm{~m} / \mathrm{s}$

when the turns are made into wind (as they are at $90^{\circ}$ ), and vice versa when turning away form the wind. While the aircraft is helped greatest by the wind at $270^{\circ}$ it is hindered at $90^{\circ}$, this creates the non-symmetrical larger peak at $90^{\circ}$, and smaller trough at $270^{\circ}$.

While it seems solely from Fig. 8 that surveying with a sweep angle of $270^{\circ}$ to the wind is best way to decrease your flight time in wind. However, there are a huge number of other parameters at play that we have not examined. As mentioned in Section II when constructing the Boustrophedon paths there are four corners that the aircraft could start from, the starting corner will change which sweep angle the upwind distance peak will be located. As the starting corner is normally the one closest to the launch point, starting at an upwind corner to minimise your flight time is not realistic. 


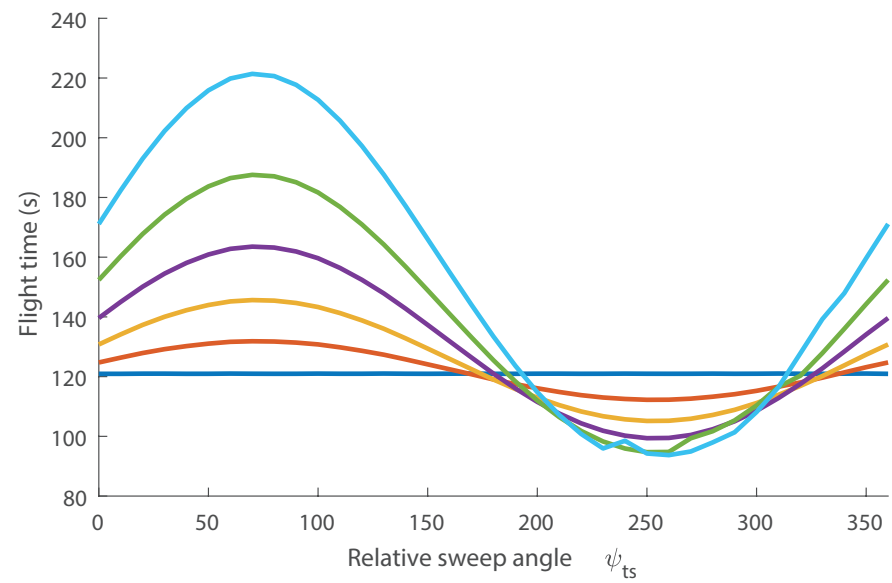

Fig. 10: Total flight time along sweep paths for $0-360^{\circ} \psi_{t s}$, at $V_{w}$ for $0-10 \mathrm{~m} / \mathrm{s}$

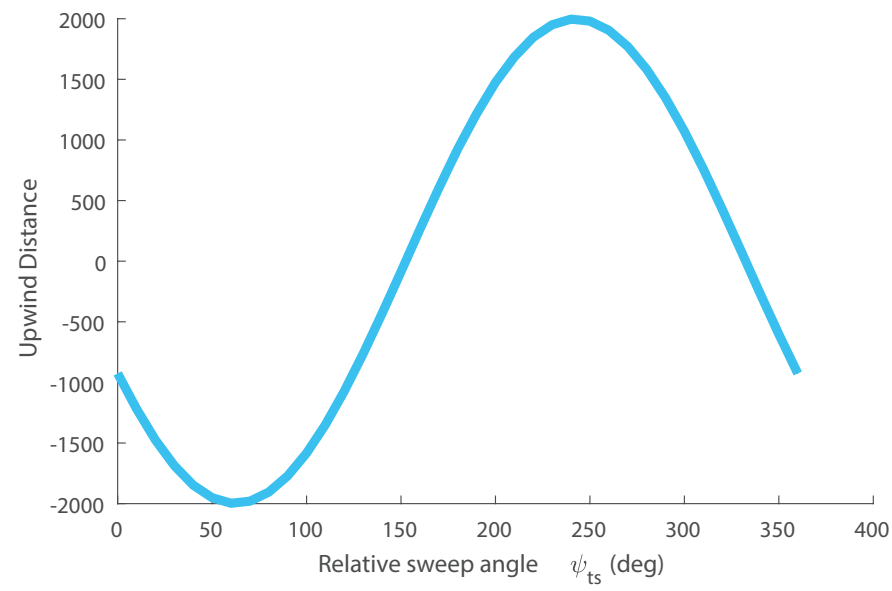

Fig. 11: The upwind distance of the survey start point to the end point

Also one must consider a more realistic survey scenario where the field to survey is a more complex concave polygon. As a concave ROI will be decomposed into smaller convex polygons this makes it even harder to use $\psi_{w}$ alone to set $\psi_{s}$ for each cell to minimise flight time. There are two main reasons for this. The first is the sweep angle that minimises the number of turns (done in previous work to minimise flight time) can directly conflict with optimum sweep angle in wind.

Secondly because a concave ROI needs to be decomposed into multiple convex cells, this mean that travelling between the cells needs to be accounted for. This is very dependant on where the initial and final waypoints are on each cell. So a small adjustment to a sweep angle to improve the flight time in wind, can dramatically increase or decrease the total travel distance. There is an example of this in Fig. 12.

\section{Simulation Results}

In order to validate the presented equations, the generated results are compared to survey paths flown in a X-Plane simulation environment. A model of a Cessna 152 is used, while this is not a UAV, it is single engined, with a cruise

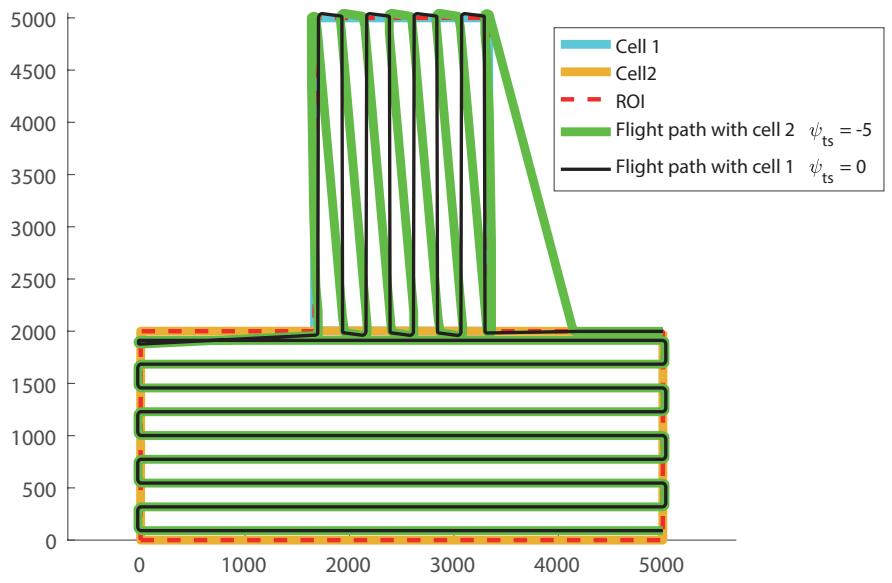

Fig. 12: Flight time comparison with $5^{\circ}$ change in sweep angle of a cell

airspeed in the same range as a larger UAV. As there is no difference between UAV and manned aircraft dynamics this is still a valid comparison.

A simple square convex ROI of $5 \times 4 \mathrm{~km}$ is chosen, the path, flight time, and velocity profiles are generated for four sweep angles: $0^{\circ}, 90^{\circ}, 180^{\circ}, 270^{\circ}$, then flown by the C-152 in X-plane with the flight parameters shown in Table 20. In order to keep the flight distance the same, the rectangular ROI is rotated $90^{\circ}$ for $\psi_{s}$ of $90^{\circ}$, and $270^{\circ}$. The calculated path, times and velocities are compared to show that this method creates an accurate enough model.

$$
\begin{array}{lll}
\psi_{w}=0^{\circ} & V=40 \mathrm{~m} / \mathrm{s} & \dot{\psi}=0.7 \mathrm{rad} / \mathrm{s} \\
V_{w}=10 \mathrm{~m} / \mathrm{s} & D_{x}=759 \mathrm{~m} & h=450 \mathrm{~m}
\end{array}
$$

The lateral controller is a simple PID controller to roll the aircraft to the angle demanded by the outer loop carrot based Pure Pursuit navigation controller laid out in [21]. The longitudinal control is performed by a total energy control system. This will control airspeed and height by controlling the ratio between total and potential energy, using throttle and elevator.

Shown in Fig. 13 is the path generated for a survey at $\psi_{s}$ of $90^{\circ}$ (as $\psi_{w}$ is 0 then $\psi_{t s}$ is still $90^{\circ}$ ). It follows the sweeps very well but the path following performance falls during the turns, where the turns are not as sharp as the generated path and there is some overshoot when rejoining the sweeps. A consequence of this is shown in Table. $\mathrm{V}$ showing that when comparing the total survey times for all four paths the flight time in X-Plane is slightly faster. This is due to the lower total flight distance as the navigation controller tended to cut corners. However this is insignificant as the error is less than $2 \%$ in all surveys.

\begin{tabular}{|c|c|c|}
\hline & \multicolumn{2}{|c|}{ Total survey time $(\mathrm{s})$} \\
\hline$\psi_{s}$ & Numerical simulation & X-Plane \\
\hline $0^{\circ}$ & $826.3 \mathrm{~s}$ & $811.9 \mathrm{~s}$ \\
$90^{\circ}$ & $772.7 \mathrm{~s}$ & $759.7 \mathrm{~s}$ \\
$180^{\circ}$ & $820.8 \mathrm{~s}$ & $804.9 \mathrm{~s}$ \\
$270^{\circ}$ & $820.8 \mathrm{~s}$ & $805.7 \mathrm{~s}$ \\
\hline
\end{tabular}

To show that the other critical factor $V_{g}$ are comparable, they are compared for all surveys in Fig. 14. It shows that 


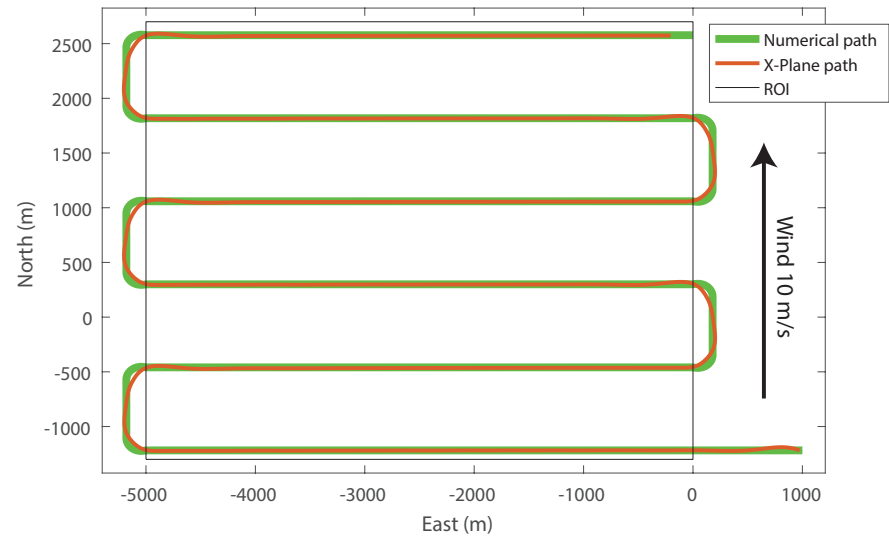

Fig. 13: Survey paths for numerical simulation compared to X-Plane flight path
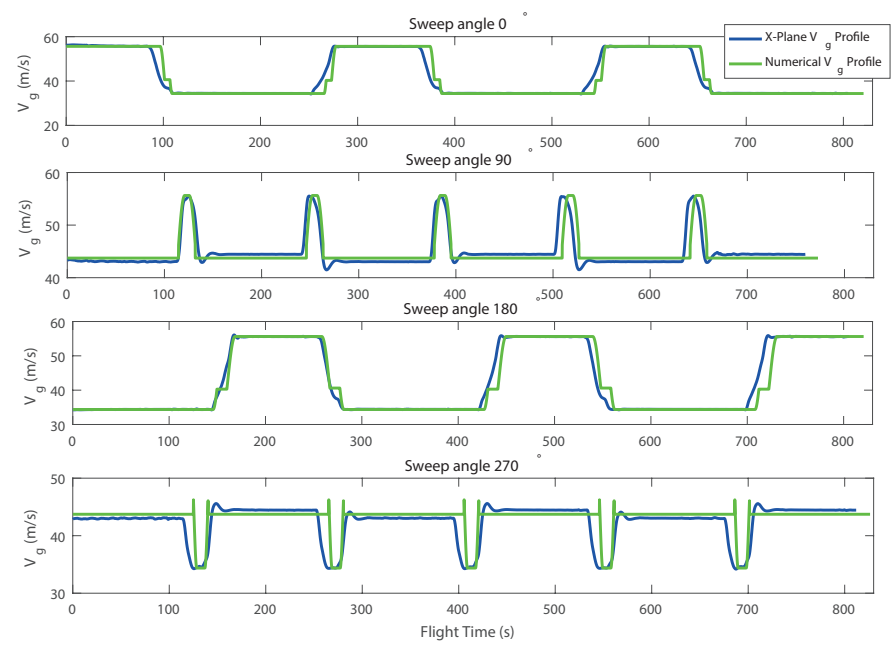

Fig. 14: Survey ground speeds profiles for numerical simulation compared to X-Plane flight path

the profiles follow each other well, but due to the slightly shorter flight distance in the X-Plane simulation there is a growing offset. In the numerically generated profiles there can be abrupt changes in the ground speed, where the $\mathrm{X}$ Plane profile is such smoother. This disparity is due to the assumption of instantaneous roll angle attainment in the trochoidal turn model. This leads to a slight under prediction of time in the turns.

\section{CONCLUSIONS \& FUTURE WORK}

In this paper we have presented a method to calculate the flight path, distance and time of an aircraft performing an aerial survey, over a convex polygon ROI in the presence of steady uniform wind. The aim was also to show that wind needs to be accounted for in BCD surveys and find out the relationship between the relative sweep angle and the flight time, in the effort to find a simple way to optimise these convex surveys.

Sweep time is predictable and minimised with sweeps perpendicular to the wind, however the method presented adds huge non-linearities that mean that there is no simple rules for which sweep angle to survey convex polygon.
When a more complex concave ROI needs to be surveyed, the transit between the decomposed cells will have much more impact on the flight times than the relation between $\psi_{t s}$ and flight time.

The model presented is shown to make accurate predictions compared to an X-Plane simulation. These accurate calculations enable full BCD optermisation to be conducted on concave ROI's, that accounts for the wind. This is to be future work.

One of the biggest potential sources for error between a real survey and the presented method, is path following accuracy. Path following accuracy is heavily dependent on the wind, for example a sweep angle perpendicular to the wind will have greater overshoot at the end of turns. To truly model the survey this needs to be factored in.

$\mathrm{X}$-Plane assumes steady uniform wind, to more rigorously test these algorithms, real life test will need to be conducted in future work.

\section{ACKNOWLEDGMENT}

This work was supported by Science and Technology Facilities Council (STFC) under Newton Fund with grant number ST/N006852/1.

\section{REFERENCES}

[1] USDA, "Farms and land in farms," United States Department of Agriculture, Tech. Rep., 2016.

[2] D. J. Mulla, "Twenty five years of remote sensing in precision agriculture: Key advances and remaining knowledge gaps," Biosystems Engineering, vol. 114, no. 4, pp. 358 371, 2013, special Issue: Sensing Technologies for Sustainable Agriculture. [Online]. Available: //www.sciencedirect.com/science/ article/pii/S1537511012001419

[3] C. Yang, J. H. Everitt, Q. Du, B. Luo, and J. Chanussot, "Using high-resolution airborne and satellite imagery to assess crop growth and yield variability for precision agriculture," Proceedings of the IEEE, vol. 101, no. 3, pp. 582-592, March 2013.

[4] D. Lamb and R. Brown, "Precision agriculture: Remote-sensing and mapping of weeds in crops," Journal of Agricultural Engineering Research, vol. 78, no. 2, pp. 117 - 125, 2001.

[5] L. Wang and J. J. Qu, "Satellite remote sensing applications for surface soil moisture monitoring: A review," Frontiers of Earth Science in China, vol. 3, no. 2, pp. 237-247, 2009. [Online]. Available: http://dx.doi.org/10.1007/s11707-009-0023-7

[6] I. Colomina and P. Molina, "Unmanned aerial systems for photogrammetry and remote sensing: A review," $\{$ ISPRS $\}$ Journal of Photogrammetry and Remote Sensing, vol. 92, pp. 79 - 97, 2014. [Online]. Available: //www.sciencedirect.com/science/article/ pii/S0924271614000501

[7] R. Gini, D. Pagliari, D. Passoni, L. Pinto, G. Sona, and P. Dosso, "Uav photogrammetry: Block triangulation comparisons," Int. Arch. Photogram. Remote Sens. Spat. Inf. Sci, vol. XL-1/W2, pp. pp.157162,2013

[8] A. Xu, C. Viriyasuthee, and I. Rekleitis, "Optimal complete terrain coverage using an unmanned aerial vehicle," in 2011 IEEE International Conference on Robotics and Automation, May 2011, pp. 2513-2519.

[9] E. M. Arkina, S. P. Fekete, and J. S. Mitchella, "Approximation algorithms for lawn mowing and milling," Computational Geometry, vol. 17 , pp. 25-50, 2000.

[10] R. N. D. Carvalho, H. A. Vidal, P. Vieira, and M. I. Ribeiro, "Complete coverage path planning and guidance for cleaning robots," in Industrial Electronics, 1997. ISIE '97., Proceedings of the IEEE International Symposium on, vol. 2, Jul 1997, pp. 677-682 vol.2. 
[11] T. Oksanen and A. Visala, "Coverage path planning algorithms for agricultural field machines," Journal of Field Robotics, vol. 26, no. 8, pp. 651-668, 2009. [Online]. Available: http: //dx.doi.org/10.1002/rob.20300

[12] C. D. Franco and G. Buttazzo, "Energy-aware coverage path planning of uavs," in 2015 IEEE International Conference on Autonomous Robot Systems and Competitions, April 2015, pp. 111-117.

[13] L. Paull, C. Thibault, A. Nagaty, M. Seto, and H. Li, "Sensordriven area coverage for an autonomous fixed-wing unmanned aerial vehicle," IEEE Transactions on Cybernetics, vol. 44, no. 9, pp. 16051618, Sept 2014.

[14] H. Choset, "Coverage of known spaces: The boustrophedon cellular decomposition," Autonomous Robots, vol. 9, no. 3, pp. 247-253, 2000. [Online]. Available: http://dx.doi.org/10.1023/A: 1008958800904

[15] Y. Li, H. Chen, M. J. Er, and X. Wang, "Coverage path planning for $\{$ UAVs $\}$ based on enhanced exact cellular decomposition method," Mechatronics, vol. 21, no. 5, pp. 876 - 885, 2011, special Issue on Development of Autonomous Unmanned Aerial Vehicles. [Online]. Available: //www.sciencedirect.com/science/article/pii/S0957415810001893

[16] J.-C. Latombe, Robot motion planning. Springer Science \& Business Media, 2012, vol. 124.

[17] G. Cai, B. M. Chen, T. H. Lee, and M. Dong, "Design and implementation of a hardware-in-the-loop simulation system for smallscale uav helicopters," Mechatronics, vol. 19, no. 7, pp. 1057 - 1066, 2009.

[18] L. E. Dubins, "On curves of minimal length with a constraint on average curvature, and with prescribed initial and terminal positions and tangents," American Journal of mathematics, pp. 497-516, 1957.

[19] L. Techy and C. A. Woolsey, "Minimum-time path planning for unmanned aerial vehicles in steady uniform winds," Journal of guidance, control, and dynamics, vol. 32, no. 6, pp. 1736-1746, 2009.

[20] M. Coombes, W.-H. Chen, and P. Render, "Landing site reachability in a forced landing of unmanned aircraft in wind," Journal of Aircraft, pp. 1-13, Feb. 2017. [Online]. Available: http://dx.doi.org/10.2514/1.C033856

[21] R. C. Coulter, "Implementation of the pure pursuit path tracking algorithm,” DTIC Document, Tech. Rep., 1992. 